\title{
Design of Heat-treated Alloy Steels Using Intelligent Multi-objective Optimisation
}

\author{
M. MAHFOUF, M. CHEN and D. A. LINKENS
}

Institute for Microstructural and Mechanical Process Engineering (IMMPETUS), Department of Automatic Control and Systems Engineering, The University of Sheffield, Mappin Street, Sheffield, S1 3JD, UK. E-mail: M.Mahfouf@shef.ac.uk

(Received on November 1, 2004; accepted on February 3, 2005)

In this paper, a new mechanism is presented to address the multi-objective optimal alloy design problem, which consists of finding the 'best' processing parameters and the corresponding chemical compositions to achieve certain pre-defined mechanical properties of steels. Fuzzy modelling has been used to establish the required mechanical properties which facilitate the Particle Swarm based multi-objective optimisation mechanism. Hence, an adaptive weighted PSO algorithm is developed to improve the performance of the standard PSO algorithm. Based on the established tensile strength and impact toughness fuzzy prediction models, the proposed algorithm has been successfully applied to the optimal design of heat-treated alloy steels. The experimental results have shown that the algorithm can locate the constrained optimal solutions quickly and provide a useful and effective guide for alloy steels design.

KEY WORDS: alloy design; multi-objective optimisation; particle Swarm; fuzzy modeling; mechanical property prediction.

\section{Introduction}

Multi-objective Optimisation (MO) problems are commonly encountered in science and engineering areas, due to the multi-criteria nature of many application problems. In complex engineering design, such as development of alloy steels, two or more, sometimes competing and/or incommensurable objective functions have to be considered simultaneously. In the development of alloy materials, the conjoint design and control of chemical composition and details of the thermomechanical processing schedules to develop optimum mechanical properties always lead to a complex exercise. The required mechanical properties of modern alloy steels are achieved by obtaining an optimum microstructure through a careful combination of alloy compositions, rolling schedules and heat treatment. In the steel industry, heat treatments (containing hardening and tempering stages) are commonly used to develop the required mechanical properties in a range of alloy steels. The mechanical properties of the material are dependent on many factors, including the tempering temperature, the quench temperature, the types of quench medium, the content of chemical compositions of the steel, the geometry of the bar, etc. Determining the optimal heat treatment regime and the required weight percentages for the chemical composites to obtain the pre-defined mechanical properties of steel is a challenge for the steel industry. To address this problem, a metal design paradigm which combines mechanical property prediction and an optimisation mechanism has been established as shown in Fig. 1. It can be seen that an optimisation mechanism consists of two important components: a reliable prediction model and an efficient optimisation para- digm. As the available physical knowledge of the heat treatment process is not enough to allow one to compute the mechanical properties, it is crucially important to establish reliable property prediction models. These will be obtained through elicited data-driven models, such as Neural Network models ${ }^{1)}$ and neural-fuzzy models. ${ }^{2)}$ These predictive models are then used to predict the mechanical properties of steel such as the Tensile Strength (TS), the Reduction of Area (ROA), Elongation and Impact Toughness. The predicted properties can be used as the objectives of the optimal metal design. In this study, the emphasis is on impact toughness and tensile strength properties oriented alloy design. Fuzzy models for the tensile strength and impact toughness prediction are used to facilitate the optimisation process for multi-objective approaches. For optimisation, the new paradigm of Particle Swarm ${ }^{3)}$ has been used as an optimisation mechanism for alloy design. This paper incorporates the two challenging problems: impact toughness prediction and multi-criteria optimisation into a unified framework and provides a useful application

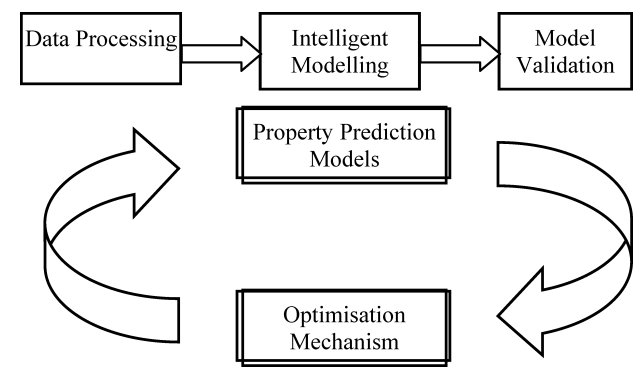

Fig. 1. Optimisation of metal design. 
example for multi-objective industrial design problems.

The remaining parts of the paper are structured as follows: Sec. 2 presents the data-driven fuzzy modelling approach for tensile strength and Charpy impact toughness prediction. Sec. 3 describes the adaptive weighted nondominated sorting PSO algorithm. In Sec. 4, multi-objective optimal alloy designs using the proposed PSO algorithm are presented. Finally, results, discussions and conclusion will be given in Sec. 5 .

\section{Fuzzy Modelling for Mechanical Property Predic- tion}

Fuzzy modelling is one of the most active research fields in intelligent computation, which combines the facility of explicit knowledge representation in the form of if-then rules (the mechanism of reasoning in human understandable terms) and the ability of approximating complicated non-linear functions. A fuzzy model is a system description with fuzzy quantities, which are expressed in terms of fuzzy numbers or fuzzy sets associated with linguistic labels. Consider a collection of $N$ data points $\left\{P_{1}, P_{2}, \ldots, P_{N}\right\}$ in a $m+1$ dimensional space that combines both input and output dimensions. A generic fuzzy model is presented as a collection of fuzzy rules in the following form:

$$
R_{i} \text { : If } x_{1} \text { is } A i_{1} \text { and } x_{2} \text { is } A i_{2} \ldots \text { and } x_{m} \text { is } A_{i m} \text { then } y=z i(x)
$$

where $\left.x=\left(x_{1}, x_{2}, \ldots, x_{m}\right)\right) \in U$ and $y \in V$ are linguistic variables, $A_{i j}$ are fuzzy sets of the universes of discourse $U_{j} \in R$, and $z_{i}(x)$ is a function of input variables. Typically, $z$ takes the following three forms: a singleton, a fuzzy set or a linear function. Fuzzy logic systems with the centre average defuzzifier, the product-inference rule and the singleton fuzzifier are of the following form:

$$
y=\sum_{i=1}^{n} z_{i}(x)\left[\prod_{j=1}^{m} \mu_{i j}\left(x_{j}\right)\right] / \sum_{i=1}^{n} \prod_{j=1}^{m} \mu_{i j}\left(x_{j}\right) \ldots \ldots . .(2)
$$

where $\mu_{i j}(x)$ denotes the membership function of $x_{j}$ belonging to the $i$ th rule.

According to the fuzzy modelling paradigm proposed by Chen and Linkens, ${ }^{2)}$ the data-driven fuzzy modelling consists of the following tasks: 1) generate an initial fuzzy rule-base from data, including initialising the membership function parameters; 2) determine the number of fuzzy rules; and 3) optimise the parameters both in the antecedent and consequent parts of the rules. All membership function parameters in the antecedent part and the linear weights in the consequent part of the fuzzy rules are optimised via a gradient-descent learning algorithm. The acquired fuzzy model should be validated under certain performance indices, such as accuracy, generality, complexity, interpretation, etc. Once the model performance achieves the pre-defined criteria, the final model is produced.

In materials engineering, it is important to establish an appropriate composition-processing-property model for materials development. Particular emphases are placed on the tensile strength and impact toughness, which are two crucially important mechanical properties in alloy steels. In recent years, various models have been developed for ten- sile strength prediction. ${ }^{4-6)}$ However, not much work has been seen on impact toughness prediction. As one of the most important characteristics of alloy steels, toughness is assessed by the Charpy V-notch impact test. The absorbed impact energy and the transition temperature defined at a given Charpy energy level are regarded as the common criteria for toughness assessment. However, a value of Charpy impact energy only allows a rather qualitative description of toughness because of its complex and subtle connection with material composition and microstructure. Recent years have seen work attempting to unravel this through Charpy impact test modelling, such as instrumented Charpy test, ${ }^{7}$ ) modelling of Charpy impact energy data using statistical analyses $^{8)}$ and numerical modelling of the ductile-brittle transition. ${ }^{9,10)}$ However, not much work has been done to date on establishing generic composition-processing-impact toughness models. In this study, a recently proposed Charpy toughness modelling approach ${ }^{11,12)}$ has been used to establish generic toughness prediction models which link materials compositions and processing conditions with Charpy impact properties for heat-treated alloy steels. To build a Charpy toughness prediction model, 3804 heat treatment process data were collected and used for modelling. The data set contains the chemical compositions, the processing parameters and the Charpy energy $C v(\mathrm{~J})$ tested at different temperatures (between -60 and $23^{\circ} \mathrm{C}$ ). $50 \%$ of the data were used for model training and the remaining $50 \%$ of the data were used as testing data. The model inputs include chemical compositions (C, Si, Mn, S, Cr, Mo, Ni, Al, V), the geometry of the bar (width and thickness) and the processing variables (Quench.T, Temp.T and Charpy test Temperature). The model output is the predicted Charpy impact energy $C v$. Based on the obtained 3804 heat treatment process data, the fuzzy model with 6 rules was developed to predict the Charpy impact energy using the fuzzy modelling procedure described in the previous section. The predicted result with a Root-Mean-Square-Error of RMSE $=18$ is shown in Fig. 2(a). Also, the tensile strength prediction model with 6 fuzzy rules has been developed in the same fuzzy modeling framework. The tensile strength prediction result with a $\mathrm{RMSE}=38$ is displayed in Fig. 2(b). Both the Charpy impact energy and tensile strength prediction models are then used for toughness assessment in optimal alloy design.

\section{Particle Swarm Algorithm for Multi-objective Opti- misation}

As already stated, alloy design remains very much a challenging multi-objective optimisation problem, which consists of finding the optimal chemical compositions and processing parameters for pre-defined property requirement. PSO is a relatively new technique for finding the optimal regions of complex search spaces via the interaction of individuals in a population of particles. It was originally introduced by J. Kennedy and C. Eberhart in 1995. ${ }^{3)}$ Unlike evolutionary algorithms, which are based on the principle of survival of the fittest, PSO is motivated by the simulation of social behaviour of flocks. As Kennedy stated, ${ }^{13)}$ the algorithm is based on a metaphor of social interaction, searches a space by adjusting the trajectories of individual 


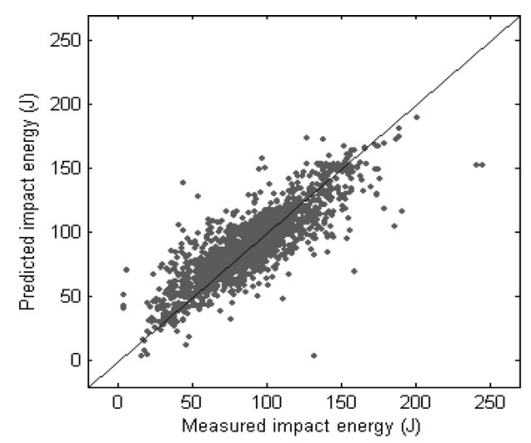

(a)

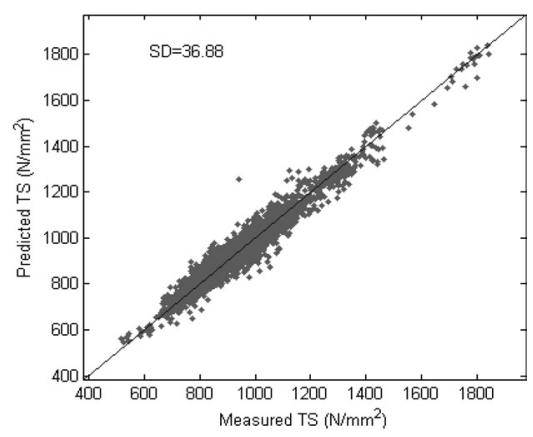

(b)

Fig. 2. Model predictions using the neural-fuzzy paradigm: (a) Ultimate Tensile Strength (UTS); (b) Charpy Impact Energy.

vectors, called "particles" as they are conceptualised as moving points in the multidimensional space. The individual particles evaluate their positions in relation to a goal at every iteration. They are drawn stochastically towards the positions of their own previous best performance and the best previous performance of their companions. The PSO algorithm has been shown to be a successful optimiser in a wide range of functions. It is easily implemented and usually results in faster convergence rates than the GA. ${ }^{13)}$ In this paper, an improved PSO algorithm is introduced to address this multi-objective alloy optimal design problem.

The PSO algorithm defines each particle as a potential solution to a problem in a dimensional space, with particle $i$ represented as: $x_{i}=\left(x_{i 1}, x_{i 2}, \ldots, x_{i d}\right), i=1,2, \ldots N$. Where $d$ is the search dimension and $N$ determines the number of particles in the population. The original formula developed by Kennedy and Eberhart was improved by Shi and Eberhart ${ }^{14)}$ by introducing an inertia weight $w$. During each iteration, the particles' position is modified according to the following equations:

$$
\begin{array}{r}
v_{i}(t)=w v_{i}(t-1)+c_{1} r_{1}\left(p_{i}-x_{i}(t-1)\right)+c_{2} r_{2}\left(p_{\mathrm{g}}-x_{i}(t-1)\right) \ldots \\
x_{i}(t)=v_{i}(t)+x_{i}(t-1) \ldots \ldots \ldots \ldots \ldots \ldots \ldots \ldots \ldots \ldots \ldots \ldots \ldots \ldots \ldots \ldots
\end{array}
$$

where $v_{i}$ is the velocity, $w$ is the inertia weight, $c_{1}$ and $c_{2}$ are positive constants, and $r_{1}$ and $r_{2}$ are random numbers obtained from a uniform random distribution function in the range $[0,1] . p_{i}$ represents the best previous position of the $i$ th particle and $p_{\mathrm{g}}$ denotes the best particle among all the particles in the population. The inertia weight $w$ plays the role of balancing the global and local searchers and its values may vary during the optimisation process. A large inertia weight encourages a global search while a small value pursues a local search. Shi and Eberhart ${ }^{15)}$ suggested changing the inertia weight linearly from 1 to 0.4 to restrict the global search ability of the PSO algorithm at the end of a run.

To improve the performance of the PSO for multi-objective optimisation problems, we proposed an Adaptive Weighted PSO (AWPSO) algorithm, ${ }^{16)}$ in which the velocity in Eq. (3) is changed as follows:

$$
v_{i}(t)=w v_{i}(t-1)+\alpha\left[r_{1}\left(p_{i}-x_{i}(t-1)\right)+r_{2}\left(p_{\mathrm{g}}-x_{i}(t-1)\right)\right]
$$

The second term in Eq. (5) can be viewed as an acceleration term, which depends on the distances between current position $x_{i}$ and personal best $p_{i}$ and global best $p_{\mathrm{g}}$. The acceleration factor $\alpha$ is defined as:

$$
\alpha=\alpha_{0}+t / N_{t} \quad t=1,2, \ldots, N_{t}
$$

where $N_{t}$ denotes the number of generations, $t$ represents the current generation, $\alpha_{0} \in[0.5,1]$ is a constant. As can be seen, the acceleration term will increase as the iteration increases, which will enhance the global search ability at the end of run and will help the algorithm to jump out of the local optimum especially in multi-modal problems.

Instead of using a linearly decreasing inertia weight, we use a random number as the inertia weight and change the inertia weight at every generation using the following formula:

$$
w=w_{0}+\left(1-w_{0}\right) r
$$

where $w_{0} \in[0,1]$ is a positive constant, $r$ is a random number uniformly distributed in $[0,1]$. In this paper we set $w_{0}=0.4$, which produces a randomly varying weight between 0.4 and 1 with a mean value of 0.7 .

To evaluate the performance of individual particles, an appropriate evaluation function should be defined. We simply use the weighted aggregation approach to construct the evaluation function $F$ for multi-objective optimization as follows:

$$
F=\sum_{i=1}^{m} \rho_{i} f_{i} ; \quad \sum_{i=1}^{m} \rho_{i}=1
$$

where $m$ is the number of objectives, $i=1,2, \ldots, m, f_{i}$ denotes the $i$ th objective function. To approximate the Pareto front instead of a certain Pareto solution, the weights $\rho_{j}$ for each objective are changed systematically and normalised as follows:

$$
\rho_{i}=\lambda_{i} / \sum_{i=1}^{m} \lambda_{i} ; \quad \lambda_{i}=U(0,1)
$$

The function $U(0,1)$ generates a uniformly distributed random number within $[0,1]$. In this way, one can obtain a uniformly distributed random weight combination, which are generated at every generation. The idea is to use dynamic weights instead of fixed weights to achieve the Pareto solutions. This dynamically weighted aggregation approach was introduced for the selection of best $p_{\mathrm{g}}$. To improve the convergence of the multi-objective optimisation, the non-dominated sorting technique, which was proposed and improved 
by Deb et al. ${ }^{17,18)}$ and then introduced into the PSO algorithm by Li X., ${ }^{19)}$ has been also used in the modified PSO algorithm. The effectiveness of the AWPSO algorithm has already been demonstrated. ${ }^{15)}$ Compared to several widely recognized evolutionary algorithms, such as NSGA II and SPEA, the proposed algorithm achieved better convergence and diversity when tested on challenging benchmark functions, such as ZDT1-ZDT4. ${ }^{20)}$

In the light of the above considerations, the proposed algorithm can be summarised as follows:

\section{1) Initialisation}

Set population number and iteration number. Initialise the position $x_{i}$ and velocity $v_{i}$ of the particles with random numbers within the pre-defined decision variable range.

\section{2) Evaluation}

Generate the random numbers $r$ and $\lambda_{l}$, and calculate the weights $w$ and $\rho_{i}$ in Eqs. (7) and (9) respectively. Evaluate each particle in the current population using Eq. (8).

\section{3) Generate new particles}

Calculate the new velocity and new position based on the current position $x_{i}$ using Eqs. (5) and (4) as well as the objective function values for all the new particles. Combine all particles together and store them in a temporary list.

\section{4) Non-dominated sorting}

a) Identify the non-dominated solutions in the previous temporary list and store them as the "Pareto front".

b) Remove the non-dominated particles from the above temporary list.

c) Identify the non-dominated solutions from those remaining in the temporary list and store them as the next "front".

d) Repeat b) and c) until all particles are ranked into successive different fronts.

5) Select the particles for next iteration

6) Calculate the objective functions values for all particles

7) If necessary go to 2

8) Otherwise, store the non-dominated solutions from the final population

\section{Multi-objective Optimal Design for Heat-treated Alloy Steels}

The proposed algorithm has been applied for the optimal design of heat-treated alloy steels. In this section, details relating to the optimisation of the Charpy impact toughness using the AWPSO algorithm are presented and discussed. The decision vector consists of the weight percentages for the chemical composites, namely: Carbon (C), Manganese (Mn), Chromium (Cr), Molybdenum (Mo), Nickle (Ni) and Tempering temperature (Temp) respectively. As aforemen- tioned, all optimisation experiments are based on the fuzzy property prediction models.

\subsection{Toughness Oriented Optimal Design}

Companies in the steel industry value highly the achievement of the required levels of toughness properties of hot rolled steel products. The optimal alloy toughness design aims at finding the appropriate chemical compositions and tempering temperature with the criterion of a minimum Charpy impact energy of $54 \mathrm{~J}$ at $-20^{\circ} \mathrm{C}$, which is equivalent to the ductile-brittle transition temperature at $54 \mathrm{~J}$ energy level which is below $-20^{\circ} \mathrm{C}$. In order to achieve the predefined toughness requirement, the model prediction error band should be taken into account in the selection of objectives. It is worth noting that the Error Band (EB), which depends on the model accuracy and training data density, provides an accurate guide to the model prediction error. The objective functions for alloy toughness design are defined as follows:

$$
\text { Minimise: } \quad f_{1}= \begin{cases}100 C v & \text { if } C v<1.5 C v_{0} \\ 2 C v_{0} / C v & \text { if } C v \geq 1.5 C v_{0}\end{cases}
$$$$
\text { Minimise: } f_{2}=\mathrm{EB}_{\mathrm{c}}
$$

Where $C v_{0}=54 \mathrm{~J}$, which stands for the required Charpy energy level, and $\mathrm{EB}_{\mathrm{c}}$ is the $95 \%$ confidence error band for the prediction model. The objective functions $f_{1}$ and $f_{2}$ represent the requirement for Charpy toughness and the corresponding model prediction error band. Figure 3 illustrates the optimisation results with the Charpy energy values against the error band corresponding to the obtained Pareto* solutions with the target value $C v_{0}=54 \mathrm{~J}$. It indicates that the two objectives are in conflict, as any improvement in one objective causes deterioration in the other. Table 1 displays different solutions selected from the Pareto front. It can be seen that the algorithm converged to a specific area that minimised the objective functions and provided optional solutions (with different combinations of chemical compositions and tempering temperature), which

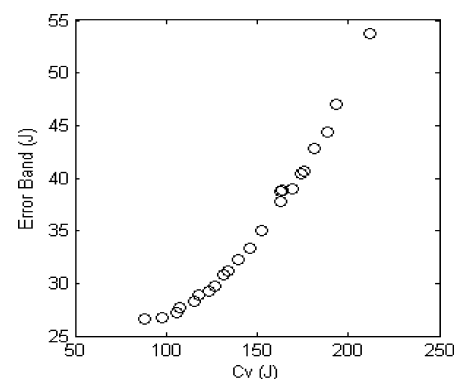

Fig. 3. Pareto-solutions for the two-objective alloy design.

Table 1. Additional solutions selected from the Pareto solutions.

\begin{tabular}{|c|c|c|c|c|c|c|c|}
\hline Solutions & $\mathrm{C}$ & $\mathrm{Mn}$ & $\mathrm{Cr}$ & Mo & Temp & $\mathrm{CV}$ & $\mathrm{EB}$ \\
\hline 1 & 0.447 & 0.811 & 1.002 & 0.291 & 697 & 88 & 26.6 \\
\hline 2 & 0.385 & 0.865 & 1.243 & 0.279 & 706 & 105 & 27.3 \\
\hline 3 & 0.337 & 0.933 & 1.044 & 0.342 & 730 & 127 & 29.7 \\
\hline 4 & 0.264 & 1.024 & 1.315 & 0.335 & 726 & 146 & 33.3 \\
\hline 5 & 0.212 & 1.197 & 1.123 & 0.420 & 725 & 169 & 39.0 \\
\hline
\end{tabular}

\footnotetext{
* Pareto solutions refer to the "best' set of solutions in the objective space.
} 
meet the pre-defined toughness requirement even when taking the error band into account.

\subsection{Optimal Alloy Design with Both Tensile Strength and Impact Toughness}

This experiment aims at finding the optimal chemical compositions and heat-treatment process parameters in order to obtain the required tensile strength while maintaining sufficient toughness. In order to achieve the pre-defined tensile strength (TS) and the corresponding 95\% confidence error band $\mathrm{EB}_{\mathrm{t}}$ for model predictions, such variables were selected as the objectives. The smaller the error band, the more reliable of the corresponding model prediction. Hence, the objectives are defined as follows:

$$
\text { Minimise: } \quad f_{3}= \begin{cases}\left|\mathrm{TS}-\mathrm{TS}_{\mathrm{t}}\right| & \text { If }\left|\mathrm{TS}-\mathrm{TS}_{\mathrm{t}}\right|<0.1 \mathrm{TS}_{\mathrm{t}} \\ 100\left|\mathrm{TS}-\mathrm{TS}_{\mathrm{t}}\right| & \text { otherwise }\end{cases}
$$

Minimise: $f_{4}=\mathrm{EB}_{\mathrm{t}}$

The first objective function $f_{3}$ indicates that the ideal solutions should be close to the target TS value $\mathrm{TS}_{t}$, the acceptable variation being $10 \%$ of $\mathrm{TS}_{\mathrm{t}}$. The biggest penalty will be assigned to the solutions that are greater than the $10 \%$ variation range of the target. Figure 4 shows the optimisation results relating to the minimisation of $f_{1}, f_{2}, f_{3}$ and $f_{4}$ defined by Eqs. (10)-(13) using the AWPSO algorithm. Figure 4(a) illustrates the TS values against the error band corresponding to the obtained Pareto solutions with the target value $\mathrm{TS}_{\mathrm{t}}=868\left(\mathrm{~N} / \mathrm{mm}^{2}\right)$. It indicates that based on the Pareto solutions, the obtained TS values are close to the target. Figure 4(b) displays the predicted Charpy impact energy values against the corresponding prediction error band. It shows that the higher Charpy energies generally correspond to the larger error bands. The trade-off between the high Charpy toughness and model reliability should be found among the obtained solutions. Table 2 shows the five different solutions selected from all the Pareto-solutions. Again, it can be seen that the optimisation method produced solutions which converged to the target together with a good spread of the Pareto-optimal solutions.

\subsection{Optimal Alloy Design with Economical Factors}

This study consists of finding the optimal chemical compositions and heat-treatment process parameters in order to obtain the required tensile strength and impact toughness while minimising the production costs. The production costs of heat-treated steels include the costs of the addition of alloying elements, such as $\mathrm{Cr}, \mathrm{Mo}, \mathrm{V}$, etc. and the costs of energy consumption during the heat-treatment process. In this experiment, five decision variables $-\mathrm{C}, \mathrm{Mn}, \mathrm{Cr}, \mathrm{Mo}$ and Tempering Temperature have been considered although other composites and temperatures could also be included. According to the contribution of the chemical composites and annealing to the cost of heat-treated steels, a new objective function to reflect such costs was introduced as follows:

$$
f_{5}=18 \mathrm{Mn}+21 \mathrm{Cr}+52.25 \mathrm{Mo} 3+4.88 \mathrm{Temp} / 600 \ldots
$$

By taking into account the above factors, the optimization experiment has been conducted based on the objective functions $f_{1}-f_{5}$, defined by Eqs. (10)-(14), using the AWPSO algorithm. Figure 5 displays the obtained paretosolutions in the objective space with the tensile strength target value $\mathrm{TS}_{\mathrm{t}}=800\left(\mathrm{~N} / \mathrm{mm}^{2}\right)$ and Charpy impact energy target value $C v_{0}=54 \mathrm{~J}$. Again, five different solutions around the tensile strength target value are selected from the Pareto-solutions and listed in Table 3. It can be seen that the optimisation method produced well spread solutions around the property targets and provided useful, practical composition and tempering temperature levels, which compromise the mechanical property requirement, the model reliability as well as the overall costs incurred.

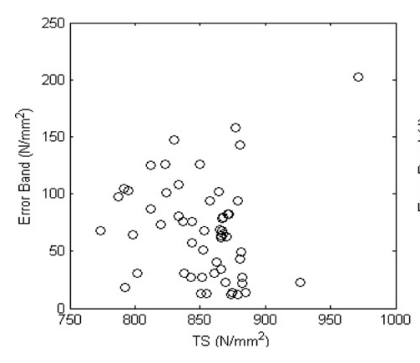

(a)

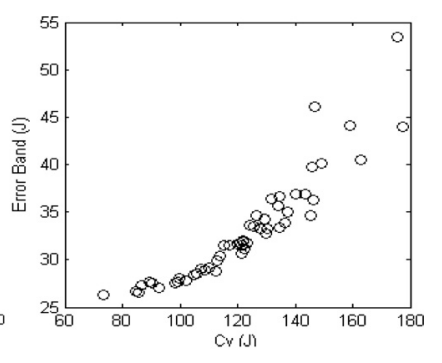

(b)
Fig. 4. Optimisation results for the four-objective alloy design: (a) Ultimate Tensile Strength (UTS); (b) Charpy Impact Energy.

Table 2. Additional solutions for the four-objective optimal alloy design.

\begin{tabular}{|c|c|c|c|c|c|c|c|c|c|}
\hline Solutions & C & Mn & Cr & Mo & Temp & TS & EBt & Cv & EBc \\
\hline 1 & 0.331 & 0.385 & 0.343 & 0.340 & 0.361 & 868 & 65.6 & 122 & 32 \\
\hline 2 & 1.022 & 0.665 & 0.895 & 0.874 & 0.786 & 873 & 12.0 & 89 & 28 \\
\hline 3 & 1.262 & 1.128 & 1.357 & 1.379 & 1.342 & 869 & 22.9 & 109 & 29 \\
\hline 4 & 0.467 & 0.353 & 0.313 & 0.377 & 0.348 & 861 & 30.4 & 110 & 29 \\
\hline 5 & 647 & 646 & 644 & 654 & 648 & 879 & 12.4 & 100 & 28 \\
\hline
\end{tabular}
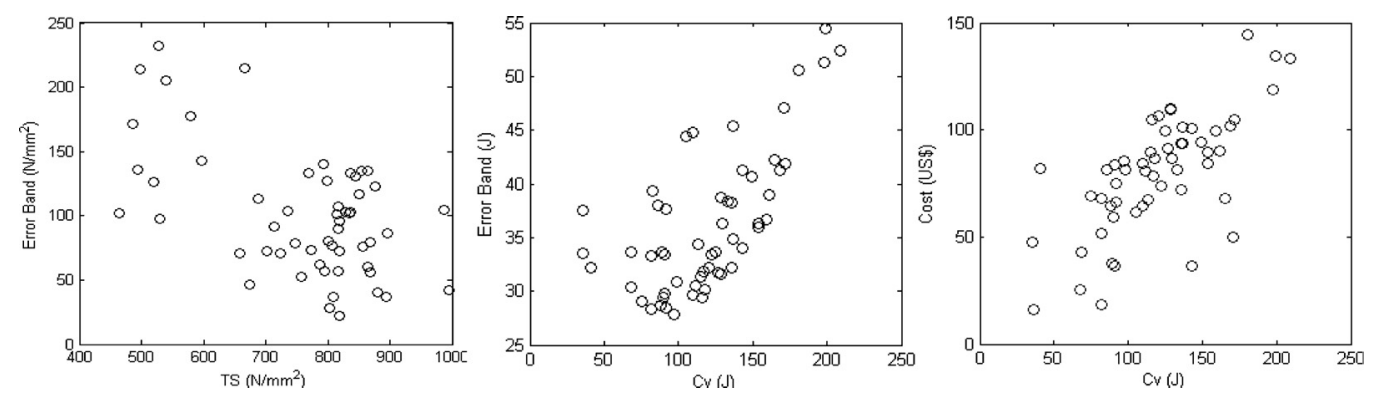

Fig. 5. Optimisation results for the five-objective alloy design. 
Table 3. Additional solutions for the five-objective optimal alloy design.

\begin{tabular}{|c|c|c|c|c|c|c|c|c|c|c|}
\hline Solutions & C & Mn & Cr & Mo & Temp & TS & EBt & Cv & EBc & Cost $\$$ \\
\hline 1 & 0.3414 & 0.8796 & 0.8063 & 0.2252 & 602 & 819 & 21.8 & 91.7 & 28.4 & 66.4 \\
\hline 2 & 0.3471 & 0.8866 & 1.3499 & 0.1442 & 651 & 809 & 36.8 & 97.4 & 27.9 & 85.5 \\
\hline 3 & 0.3439 & 0.8414 & 0.5582 & 0.3078 & 592 & 803 & 28.1 & 89.8 & 29.4 & 59.5 \\
\hline 4 & 0.3752 & 0.8387 & 1.1188 & 0.0107 & 640 & 794 & 56.4 & 81.9 & 28.3 & 67.8 \\
\hline 5 & 0.2983 & 0.9848 & 1.2023 & 0.2521 & 655 & 817 & 57.2 & 117.8 & 30.1 & 86.7 \\
\hline
\end{tabular}

\section{Conclusions}

A multi-objective optimisation mechanism using fuzzy modelling and Particle Swarm Optimisation techniques has been successfully applied to the optimal design of heattreated alloy steels. The main aim of the research was to determine the optimal heat treatment regime and the required weight percentages for the chemical composites to obtain the desired mechanical properties of steel. Based on the data-driven fuzzy model, the Tensile Strength and Charpy impact toughness can be predicted effectively and then used to facilitate optimal alloy design. The experimental results have shown that the optimisation algorithm can locate the constrained minimum design with very good convergence, and also provide a range of optional solutions which fit the pre-defined property requirement while maintaining reasonable production cost. The simulations also indicate that the algorithm produced very consistent solutions and can be effectively used in other industrial optimisation problems. Further investigations on multi-objective optimal alloy design involving more mechanical property criteria, such as Elongation, Reduction of Area, etc. will be beneficial for the steel industry as a whole.

\section{Acknowledgement}

The authors would like to thank the reviewers for their comments and suggestions which helped to improve the quality of this paper, and they would also like to thank the UK Engineering and Physical Sciences Research Council
(EPSRC) for their financial support via the IMMPETUS Phase II Programme Award, under Grant GR/R70514/01.

\section{REFERENCES}

1) J. Tenner, D. A. Linkens, P. F. Morris: Ironmaking Steelmaking, 28 (2001), 15.

2) M-Y. Chen and D. A. Linkens: IEEE Transactions on Systems, Man and Cybernetics, Part B, 31 (2001), 781.

3) J. Kennedy and R. Eberhart: Proc. of the 4th IEEE Int. Conf. on Neural Networks, IEEE, New York, (1995), 1942.

4) P. D. Hodgson: J. Mater. Process. Technol., 60 (1996), 27.

5) C. Chen, Y. Cao and S. R. LeClair: J. Alloys Compounds, 279 (1998), 30.

6) B. R. Bakshi and R. Chatterjee: J. Alloys Compounds, 279 (1998), 39.

7) M. Tahar, R. Piques and P. Forget: Proc. of the 11th Biennial European Conf. on Fracture-ECF 11, Vol. 3, Eng. Mater. Advisory Services, Warley, UK, (1996), 1945.

8) R. Moskovic and P. E. J. Flewitt: Metall. Mater. Trans. A, 28A (1997), 2609.

9) A. Needleman and V. Tvergaard: Int. J. Fract., 101 (2000), 73.

10) M. T. Todinov, M. Novovic, P. Bowen and J. F. Knott: Mater. Sci. Eng. A, A287 (2000), 116.

11) M.-Y. Chen, D. A. Linkens and A. Bannister: Mater. Sci. Technol., 20 (2004), 627.

12) M.-Y. Chen , D. A. Linkens, D. J. Howarth and J. H. Beynon: ISIJ Int., 44 (2004), 1108.

13) J. Kennedy: Proc. Int. Conf. on Evolutionary Computation (ICEC'97), IEEE, New York, (1997), 303.

14) Y. Shi and R. Eberhart: Proc. IEEE Int. Conf. on Evolutionary Computation, IEEE, New York, (1998), 69.

15) Y. Shi and R. Eberhart: Proc. of the 1999 Cong. on Evolutionary Computation, CEC'99, IEEE, New York, (1999), 1945.

16) M. Mahfouf, M.-Y. Chen and D. A. Linkens: Lecture Notes in Computer Science, 3242 (2004), 762.

17) K. Deb, S. Agrawal, A. Pratap and T. Meyarivan: NSGA-II, Proc. of Parallel Problem Solving from Nature-PPSN VI, Springer, (2000), 849.

18) K. Deb and T. Goel: Lecture Notes in Computer Science, 1993 (2001), 67.

19) X. Li: Lecture Notes in Computer Science, 2723 (2003), 37.

20) K. Deb: Multi-objective Optimization Using Evolutionary Algorithms, Wiley, New York, Chichester, (2001). 